\title{
Genetic Algorithm (GA) Based Optimal Feedback Control Weighting Matrices Computation
}

\author{
Abdulla I. Abdulla ${ }^{1}$ ， Jamal M. Ahmed ${ }^{2}$, Salih M. Attya ${ }^{3}$ \\ College of Electronics Engineering / University of Mosul /Mosul, Iraq \\ $\underline{\text { abd_63om@yahoo.com }}{ }^{1}, \underline{\text { Jamal1961eng@yahoo.com }}{ }^{2}, \underline{\text { salih.attya@yahoo.com }}$
}

\begin{abstract}
Linear Quadratic Regulator (LQR) is one of the most interesting control techniques adopted as a control strategy in state feedback. These types of techniques achieve good results but suffer from the problem of trial and error involved in the computation of weight matrices. The trial and error technique leads to hard tuning of the LQR controller parameters. This of course will lead to difficulty in reaching the optimal system performance.

The paper attempts to solve the above difficulty via the selection of the LQR weight matrices using Genetic Algorithm GA. This proposed solution will avoid the trail and error involved in the state feedback technique. The proposed solution has been adopted in the design of position controller of a robot arm and the results of computer simulation have shown that the proposed solution fulfill specifications, for minimum overshoot, settling and rising times.
\end{abstract}

Keywords: Robot Arm, Linear Quadratic Regulator (LQR) Genetic Algorithm(GA)

$$
\begin{aligned}
& \text { الخوارزمية الجينية المعتمدة لمسيطر التغذية العكسية المثالي لحساب مصفوفات الوزن }
\end{aligned}
$$

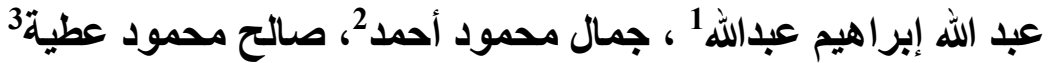

$$
\begin{aligned}
& \text { كلية هندسة الاكترونيات /جامعة الموصل/ الموصل /العراق إحل } \\
& \text { اخلاصة }
\end{aligned}
$$

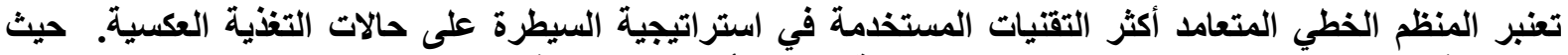

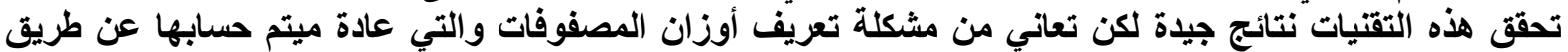

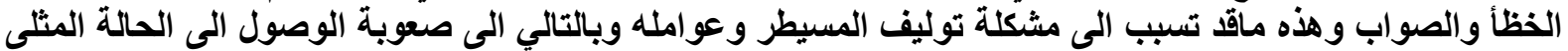

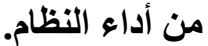
البحث بعرض تصميم مسيطر لموقع الذراع الآلي بواسطة اختيار معاملات المنظم الخطي المتعامد بالخوارزمية الجينية.

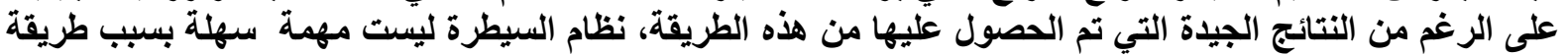

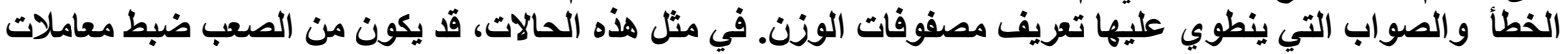

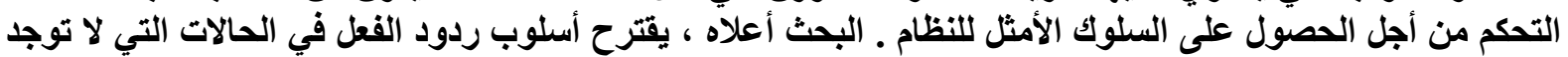

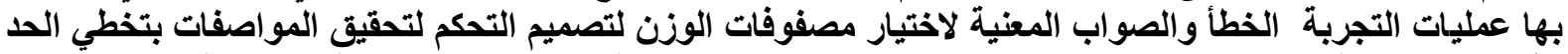

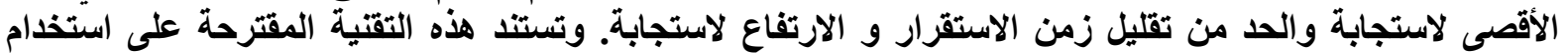

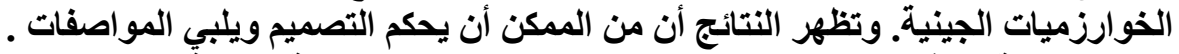

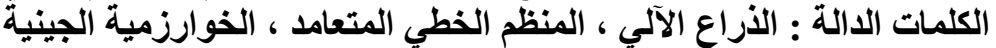




\section{1-Introduction}

In general, the arm of a robot has a number of joints. The current approach to the design of control systems for robot joints is to treat each joint of the arm as a simple joint servo mechanism ,ignoring the effect of the movements of all other joints. In industrial robots, hydraulic or pneumatic actuators may be used rather than dc servomotors. Due to it's excellent speed and position control characteristic ,the dc servomotors has been widely used in industry ,therefore the actuator is assumed to be an armature-control dc motor . In addition it is assumed that the robot arm is connected to the motor through gears. "The control systems performance depends on the parameters calculated through the use of optimization techniques. The most used techniques for state feedback control design are the Linear Quadratic Regulator (LQR) “ [1]. The LQR optimal feedback is one of many tools that can be used to improve the stability performance of a position control of a robot arm. Using LQR theory, it has been established in control linear system, a set of optimal feedback gains may be found using minimization of a quadratic index and makes it in a stable closed-loop system. The problem in LQR application is adjustment to determine the elements of weighting matrix $\mathrm{Q}$ and $\mathrm{R}$. Conventional methods use many trial-error actions to design diagonal matrices $\mathrm{Q}$ and $\mathrm{R}$ to give optimal feedback gain to the system. Therefore, Genetic Algorithm (GA) can be used to compute the weight matrices instead of the conventional trial and error used. This will give better tuning of the controller and gives optimum controller design. Therefore the aim of the paper is to investigate the possibility of using GA controller method in the computation of the weight matrices of the LQR contrller.

\section{2- Robot Arm Mathematical Model}

Figure 1 illustrates a single-joint robot arm. The block diagram of the robot joint control system is as shown in Figure (2) [2]. The robot arm is not affected by gravity and rigid. The dynamic behavior of the robot arm control system is given by the following equations [3].

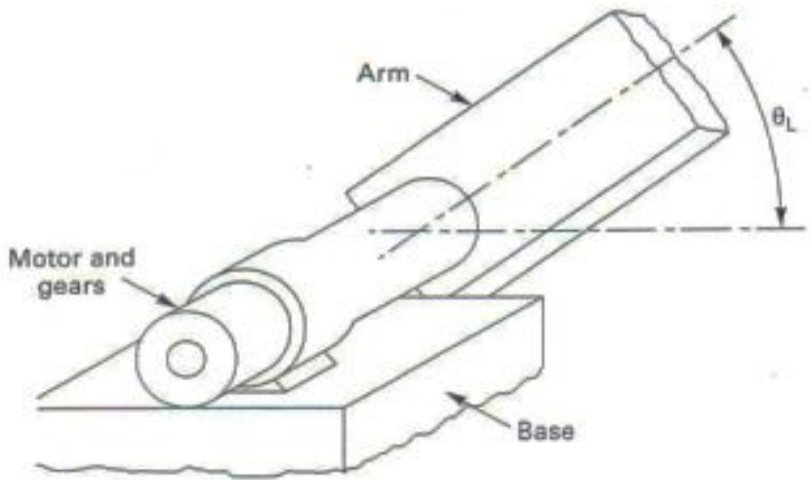

Fig. (1). Single-Joint robot arm

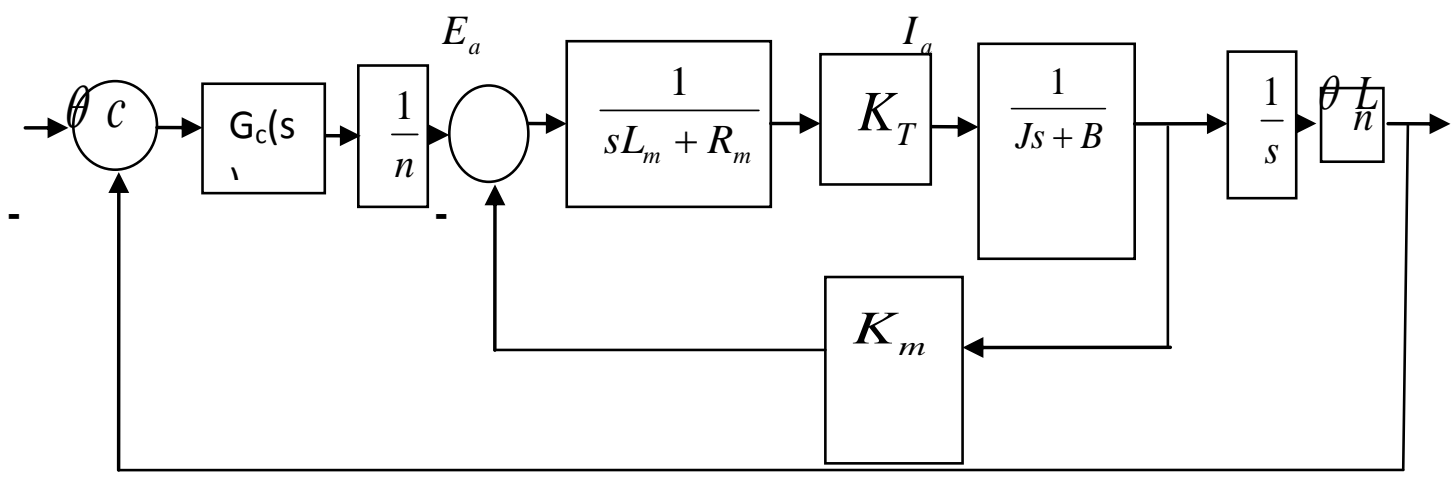

Fig. (2). Robot Joint Control System 


$$
\begin{aligned}
& e_{a}(t)=R_{m} i_{a}(t)+L_{m} \frac{d i_{a}(t)}{d t}+e_{m}(t)---------------(1) \\
& e_{m}(t)=K_{m} \frac{d \theta_{m}(t)}{d t}-----------------------(2) \\
& T_{m}=K_{T} i_{a}(t)---------------------------(3) \\
& T_{m}=J \frac{d^{2} \theta_{m}(t)}{d t^{2}}+B \frac{d \theta_{m}(t)}{d t}--------------------(4) \\
& J=J_{m}+n^{2} J l--------------------------(5) \\
& B=B_{m}+n^{2} B l--------------------------(6) \\
& \theta_{L}=n \theta_{m}-------------------------(7)
\end{aligned}
$$

After simplification and taking the ratio of $\theta_{L}(s) / E_{a}(s)$ we will get the transfer function as below.

$$
\frac{\theta_{L}(s)}{E_{a}(s)}=\frac{K_{T} n}{J L_{m} S^{3}+\left(R_{m} J+B L_{m}\right) S^{2}+\left(K_{T} K_{m}+R_{m} B\right) S}
$$

where.

$R_{m}=$ armature- winding resistance in ohm.

$L_{m}=$ armature - winding inductance in Henry.

$i_{a}=$ armature - winding current in ampere.

$e_{a}=$ armature voltage in volt.

$e_{m}=$ back emf in volt.

$K_{m}=$ back emf constant in volt / $(\mathrm{rad} / \mathrm{sec})$

$T_{m}=$ torque developed by the motor in N.m

$K_{T}=$ motor torque constant in N.m/A

$J=$ moment of inertia of motor and robot arm in $\mathrm{kg} . \mathrm{m}^{2} / \mathrm{rad}$.

$B=$ viscous - friction coefficient of motor and robot arm in N.m/rad $/ \mathrm{sec}$.

$\theta_{m}=$ angular displacement of the motor shaft in rad.

$\theta_{L}=$ angular displacement of the robot arm in rad.

$\theta_{c}=$ angular displacement of the reference input in rad.

$n=$ gear ratio $N_{1} / N_{2}$

The robot arm control system under study has the following parameters.

$R_{m}=21, L_{m}=2, K_{T}=38, J=2, B=1, K_{m}=0.5$ and $n=1 / 20$. 
The block diagram of the servo control system for one of the joint of a robot is shown in Fig(3).[3]

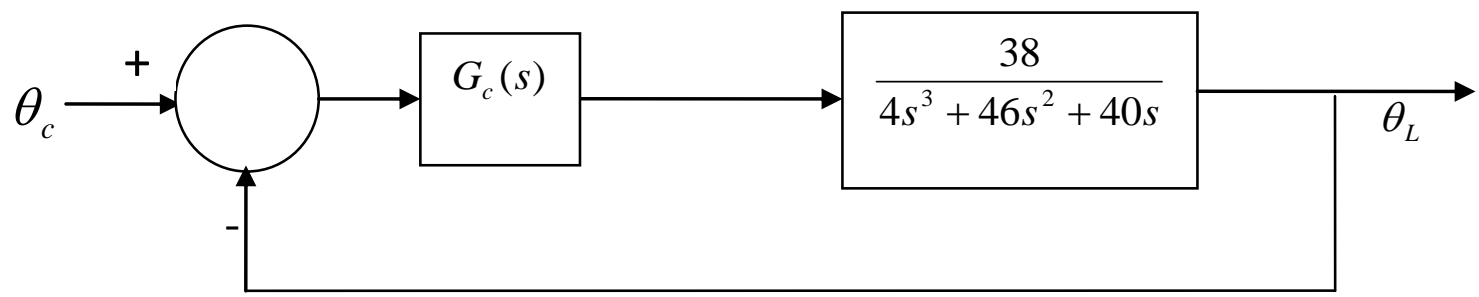

Fig. (3) Joint Control System For A Robot Arm

\section{Adopted Control Methods}

The control methods adopted in this research are Linear Quadratic Regulator (LQR) and the genetic algorithm (GA). The controller specifications adopted in the design are;

1) Overshot percentage should be less than $10 \%$.

2) Rise time (Tr) should be less than 4 sec.

3) Settling time (Ts) should be less than 6 second.

4) Steady-state error should be less than 0.005

\subsection{LQR Controller Method}

LQR is one of the modern control methods that provide the best possible performance with respect to some given measure. LQR uses statespace approach which is relatively simple to work with a multi-output system. For the brief description of the LQR controller, the full state feedback representation of robot arm system has been adopted. The structure of the LQR controller is as shown in Fig. 4.

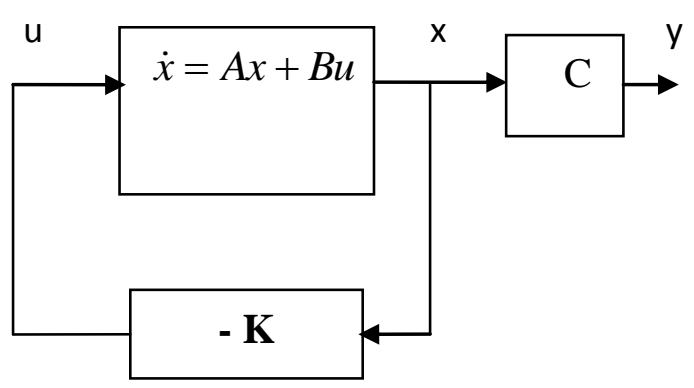

Fig. 4 LQR controller structure

The robot arm can be described by the following state and output matrix equations [7]

$$
\begin{aligned}
& \dot{x}(t)=A x(t)+B u(t) \\
& y(t)=C x(t)+D u(t)
\end{aligned}
$$

It is worth to mention here that all the three states of $\mathrm{x}$ are available for the controller. The feedback gain of the optimal control is given by the following vector $\mathrm{V}$ equation

$$
\begin{aligned}
& K=\left[\begin{array}{llll}
K_{1} & K_{2} & K_{3}
\end{array}\right] \\
& ----------------- \text { (10) } \\
& u(t)=-K x(t) \\
& ------------------ \text { (11) }
\end{aligned}
$$

This need to be selected in such away that minimizes the performance index, $\mathbf{J}$;

$$
J=\int_{0}^{\infty}\left(x^{T} Q x+u^{T} R u\right) d t
$$


where $\mathrm{Q}$ is state-cost matrix and $\mathrm{R}$ is performance index matrix. The index $\mathrm{J}$ need to be minimzed when;

$\mathrm{K}=\mathrm{R}^{-1} \mathrm{~B}^{\mathrm{T}} \mathrm{P}$

where $\mathrm{p}$ is an $n \times n$ matrix determined from the solution of the following equation

$A^{T} P+P A-P B R^{-1} B^{T} P+Q=0$

Trial and error need to be adopted in the selection of the two matrices $\mathrm{Q}$ and $\mathrm{R}$. The selection of large matrix $Q$ suggest that the performance index $\mathrm{J}$ is small. However, the selection of large matrix $\mathrm{R}$ means that the control input $\mathrm{u}$ must be smaller to keep performance index $\mathrm{J}$ small. One should select $\mathrm{Q}$ to be always positive or zero at each time t. And the scalar quantity $\mathrm{Ru}^{2}$ is always positive positive semi definite and $\mathrm{R}$ to be positive definite. This means that the scalar quantity $\mathrm{x}^{\mathrm{T}} \mathrm{Q} \mathrm{x}$ is at each time $\mathrm{t}$ eq.(14) can be easily programmed for a computer, or solved using MATLAB eq.(14) is often called the Riccati Equation. This optimal control called the Linear Quadratic Regulator (LQR) which is shown in state-space configuration in Fig. 4.Combine Fig.3 with Fig.4 yields Fig.5 which shows the use of LQR controller with the Robot Arm [4].

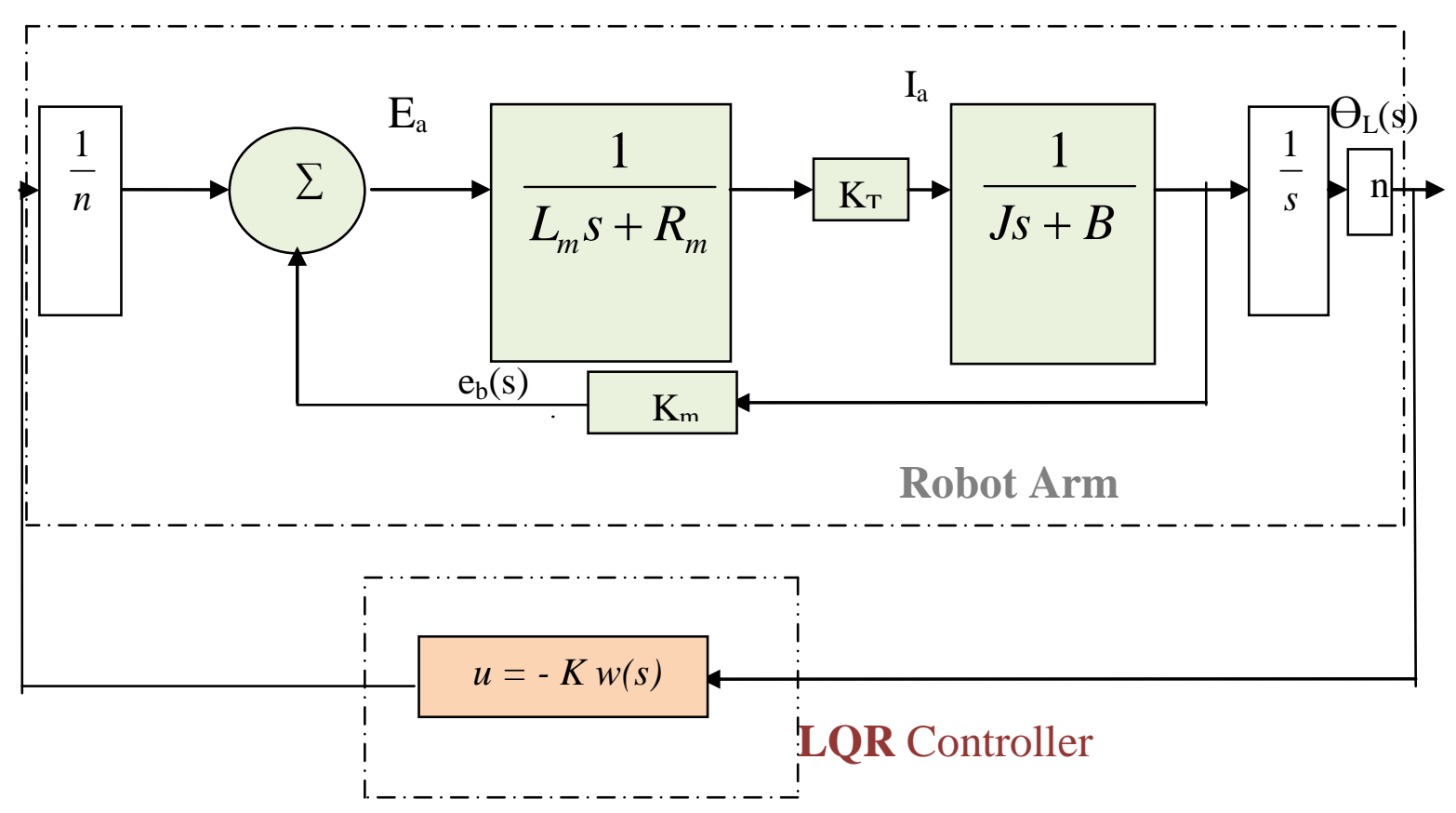

Fig. 5- Robot Arm System with LQR

\subsection{Genetic Algorithm Method}

The second type of the contrllor methods adopted is the Genetic Algorithms (GA's). It is a stochastic search method that mimics the process of natural evolution. GA begins usually with no knowledge of the correct solution. It relies entirely on responses from its environment and evolution operators (i.e. reproduction, crossover and mutation) to arrive at the best solution. GA avoids local minima and converging to sub optimal solutions. This can be achieved via starting at several independent points and searching in parallel. This capability allows the GAs to locate high performance areas in complex domains without any problem with high dimensionality. The typical inititilization of the GA process is with a 
random population that formed by a real-valued number or a binary string called a chromosome. The fitness of each chromosome is assessed and a survival of the fittest strategy is applied. There are three main stages of a GA. The procedure used with GA controller method has three stages, reproduction, crossover and mutation. This procedure can be described briefly with following sequence steps [ 5].

Step 1: Generate initial population randomly

Step 2: Evaluate fitness of the population

Step 3: Select parents from population

Step 4: Apply crossover operation on parents

Step 5: Apply mutation operation on parents

Step 6: Evaluate fitness of the population

Step 7: Checking convergence

Step 8: If convergence is achieved stop else go to step 3

\section{Controller Objective Function}

The objective function is essential for both controller methods LQR and GA methods. With the LQR method the objective function is used to evaluate the best LQR controller for the DC motor system. Specifically, it can be created to determine the LQR controller that gives the highest overshoot, fastest rise time or fastest settling time. However, all these aspects of controller objective can be combined with an objective that minimizes the error of the controlled system response.

With the GA the specification of an objective function is a difficult task. This is because, "each chromosome in the population is passed into the objective function at a time. The chromosome is then evaluated and assigned a number to represent its fitness, the bigger its number the better its fitness. The genetic algorithm uses the chromosome's fitness value to create a new population consisting of the fittest members. The chromosome is formed by three values that correspond to the three gains of the weight matrix $\mathrm{Q}$ and $\mathrm{R}$ to be adjusted in order to achieve a satisfactory behavior" [6].

Fig. 6 shows how these chromosomes can be defined together with the gains $q_{11}, q_{22}$ and $r_{11}$ of the $\mathrm{Q}$ and $\mathrm{R}$ weight matrices. These gains should be positive numbers and will be used to characterize the individual to be evaluated.

\begin{tabular}{|l|l|l|l|}
\hline$q_{11}$ & $q_{22}$ & $q_{33}$ & $r_{11}$ \\
\hline
\end{tabular}

Fig.6 . Chromosome Definition

The objective function is the calculation of its associated fitness. The fitness function is the measure of the quality of chromosome and can be defined as.

Minimize $\mathbf{J}$

Where,

$$
J=\frac{1}{N} \sum_{i=1}^{N} e_{i}
$$

$\mathrm{N}=3$ and 
$\mathrm{e}_{\mathrm{i}}=\left\{\mathrm{t}_{\mathrm{s}}^{2}, \mathrm{t}_{\mathrm{r}}^{2}, \mathrm{D}^{2}\right\} \quad$, where,

$\mathrm{D}=\left\{\begin{array}{l}\left(\mathrm{M}_{\mathrm{p}}-1\right) \text { if }\left(\mathrm{M}_{\mathrm{pcl}}-1\right)^{2}<0.05 \\ \infty\end{array}\right.$

$t_{s}$ is the settling time of GA, $t_{r}$ is the rising time of GA and $M_{p}$ is the maximum over shoot For $\mathrm{GA}, \mathrm{M}_{\mathrm{pcl}}$ is the maximum over shoot for closed loop response. The robot arm controller that combines the LQR with GA controller is as shown in Fig. 7.

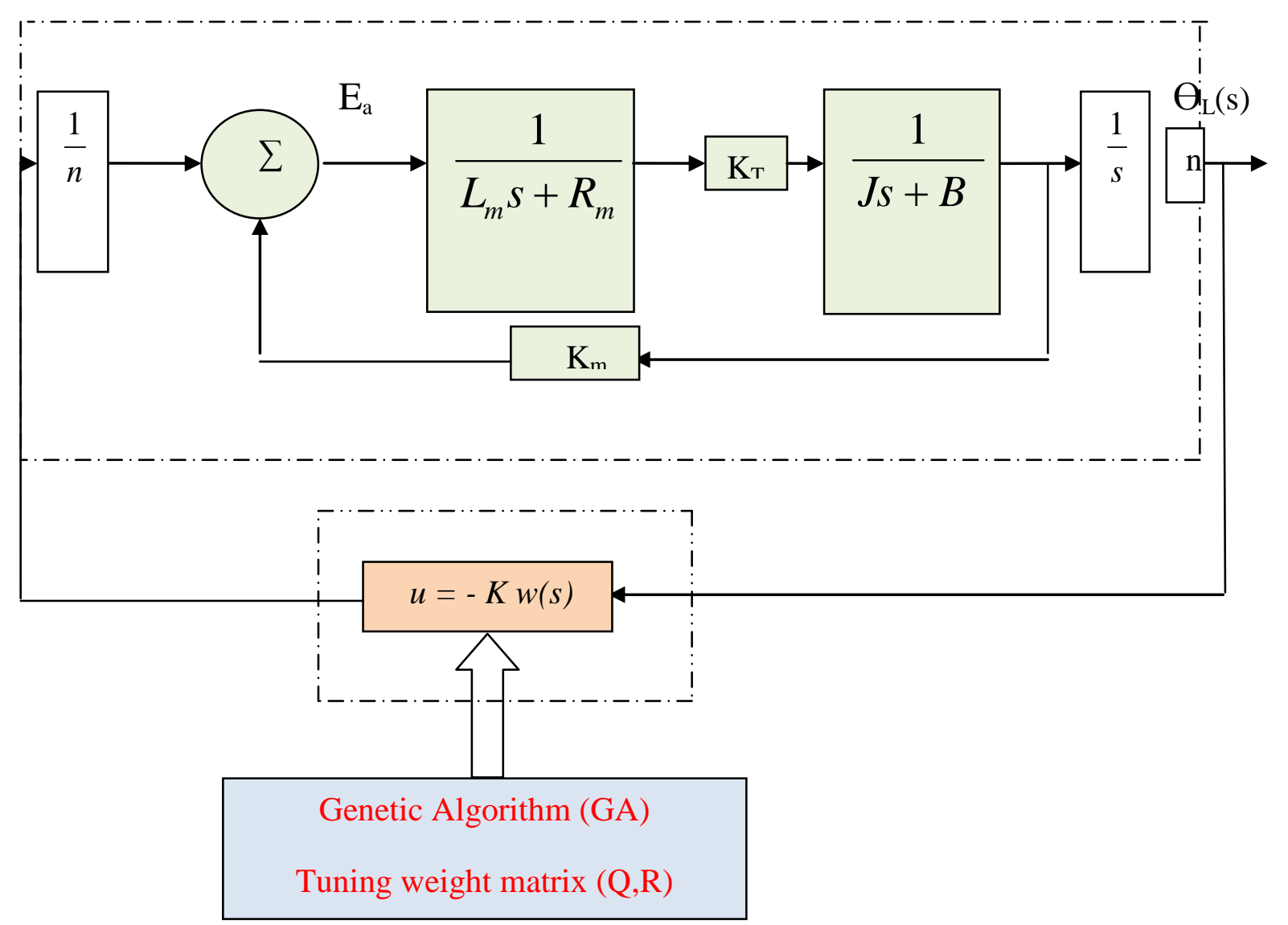

Fig. 7 Block Diagram Of GA With LQR Controller Of The Robot Arm.

\section{Simulation and Results}

The best LQR controller parameters are $Q=\operatorname{dig}(100,20,90), R=1$. The elements of $\mathrm{K}$ vector obtained by $L Q R$ method are $K=\left[\begin{array}{lll}4.4165 & 10.5425 & 9.4868\end{array}\right]$ with an Eigen values of [-14.5494, - 0.6835+i0.4299 and - 0.6835-i0.4299]. These LQR parameters for the LQR controller method give a time response for the closed loop system as shown in Fig.8.

Simulation with the GA controller method shows that the best parameters are $Q=\operatorname{dig}(5,30,180), \quad R=2$. The elements of $\mathrm{K}$ vector obtained by the GA method are $\mathrm{K}=$ [0.8334 8.6818 9.468] with an Eigen values of [-10.6652 -0.8341+i0.4462 -0.8341i0.4462]. These LQR parameters for the LQR controller method give a time response for the closed loop system as shown in Fig. 9. 


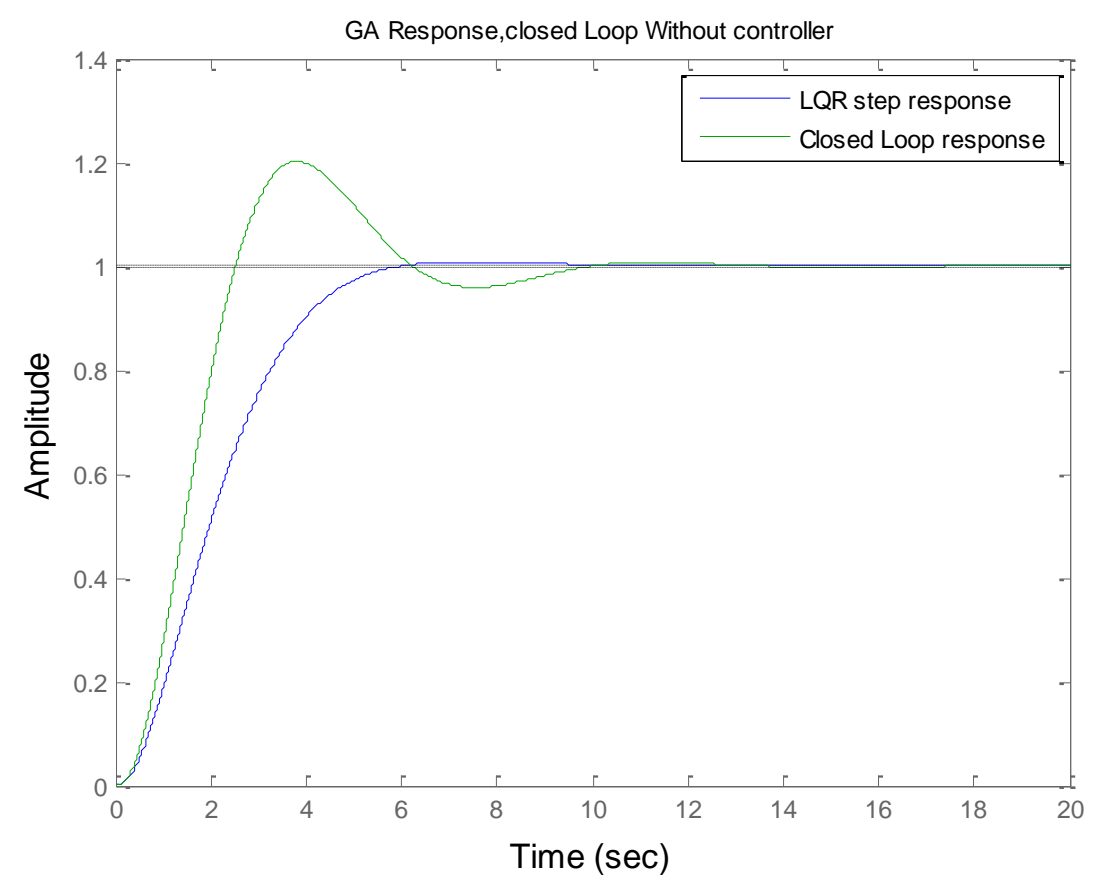

Fig. 8 Closed Loop System With The Simulated LQR Controller

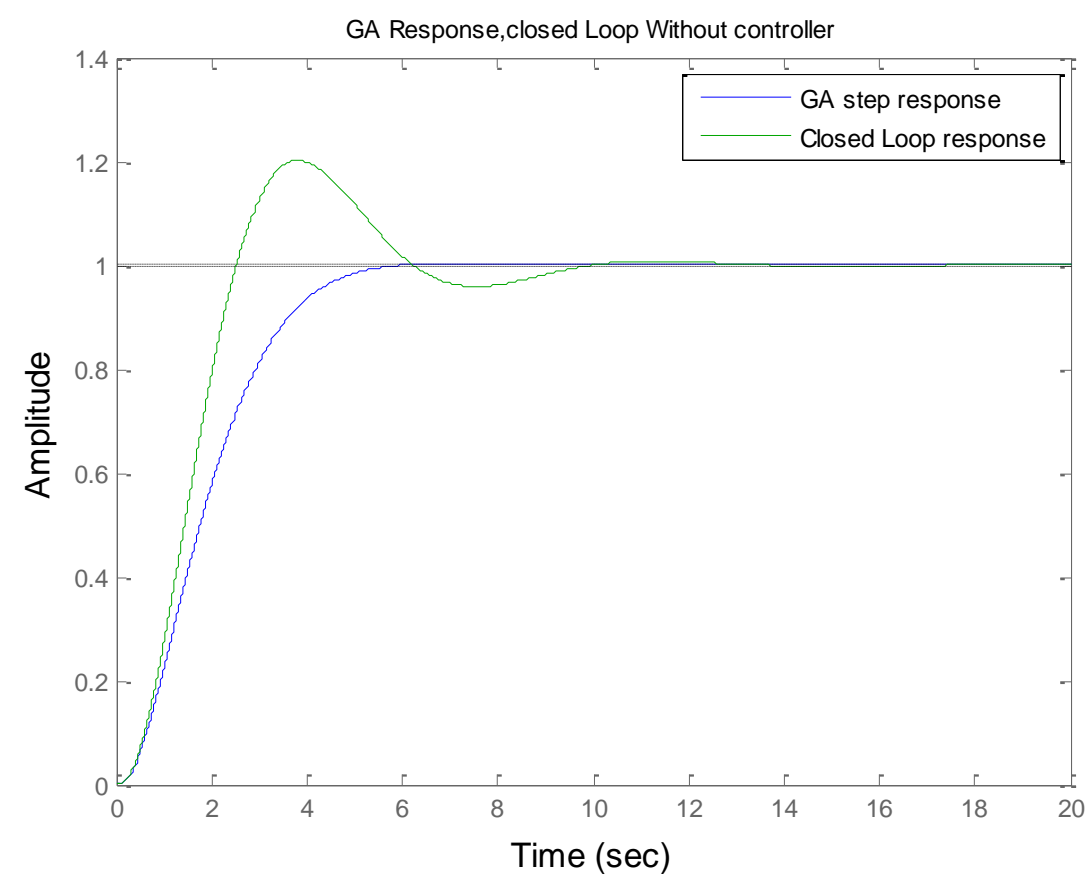

Fig. 9 Closed Loop System With The Simulated GA Controller

Finally, from the results presented in Figures 8 and 9, the overshoot time, settling time and the steady-state error have been computed for the three different cases closed loop controller status, LQR controller status and the LQR tuned with the GA asshown in Table 1. 
Abdulla: Genetic Algorithm (GA) Based Optimal Feedback Control Weighting ---

Table 1

\begin{tabular}{|c|c|c|c|}
\hline Controller Status & $\begin{array}{c}\text { Over Shoot } \\
\mathrm{Mp} \%\end{array}$ & $\begin{array}{c}\text { Settling Time ts } \\
(\mathrm{sec})\end{array}$ & $\begin{array}{c}\text { Steady-state error } \\
\text { Ess }\end{array}$ \\
\hline Closed Loop & 20.2 & 8.92 & 0 \\
\hline LQR Manual & 0.676 & 5.22 & 0 \\
\hline $\begin{array}{c}\text { LQR Tuned By } \\
\text { GA }\end{array}$ & 0.259 & 4.91 & 0 \\
\hline
\end{tabular}

\section{Conclusions}

The paper adopts two controller methods LQR and GA methods. Both methods have been applied to the state feedback control system of robot arm and their performance compared. The adoption of the GA overcomes the problem with LQR of weight matrices computation. Thus the GA has been used to solve this problem via the tuning of the LQR method.

The paper shows that when the GA is used to tune the LQR matrices an optimum dynamic response can be achieved. In according to the obtained result, it can be noted that the proposed combination of LQR and GA gives a satisfactory response of minimum overshoot and accommodation time. This is because the proposed method has been designed based on this specification of the system time response.

\section{References:}

[1]Neenu Thomas ,Dr. P. Poongodi "Position Control of DC Motor Using Genetic Algorithm Based PID Controller" Proceedings of the World Congress on Engineering 2009 Vol II WCE 2009, July 1 - 3, 2009, London, U.K.

[2] Chun Htoo Aung, Khin Thandar Lwin, and Yin Mon Myint" Modeling Motion Control System for Motorized Robot Arm using MATLAB"World Academy of Science, Engineering and Technology 42, 2008

[3] L.phillips and D.Harbar Feedback Controller System , $3^{\text {rd }}$ edition , Prentice Hall, 1996

[4] Nuraddeen Magaji, Mukhtar F. Hamza, Ado Dan-Isa" Comparison Of GA And Lqr Tuning Of Static Var Compensator For Damping Oscillations" international Journal Of Advances In Engineering \& Technology, Jan 2012

[5] P. S. Oliveira, L. S. Barros and L. G. de Q. Silveira Júnior, "Genetic Algorithm Applied to State Feedback Control Design" 2010 IEEE/PES Transmission and Distribution Conference and Exposition: Latin America

[6] G. Wang, M. Zhang, X. Xu, C. Jiang, "Optimization of Controller Parameters Based on The Improved Genetic Algorithms," Proceedings of the 6th World Congress on Intelligence Control and Automation, June 21-23, 2006, Dalian, China

[7]Ruba M.K. Al- Mulla Hummadi " Simulation Of Optimal Speed Control For A DC Motor Using Linear Quadratic Regulator(LQR) " Journal Number 3 Volume 18 march 2012 of Engineering.

[8] Imam ROBANDI, Katsumi NISHIMORI, Ryo NISHIMURA, Naganor ISHIHARA "Full-element weighting matrices Q and R design in optimal Load Frequency Control solution using genetic algorithm" flsi.cird.or.jp/iizuka2000/PAPERS/A_1_4.PDF

The work was carried out at the college of Engineering. University of Mosul 